\title{
Small values of the maximum for the integral of fractional Brownian motion
}

\author{
G. Molchan ${ }^{* \dagger} \quad$ A. Khokhlov ${ }^{\dagger}$
}

\begin{abstract}
We consider the integral of fractional Brownian motion (IFBM) and its functionals $\xi_{T}$ on the intervals $(0, T)$ and $(-T, T)$ of the following types: the maximum $M_{T}$, the position of the maximum, the occupation time above zero etc. We show how the asymptotics of $P\left(\xi_{T}<1\right)=p_{T}, T \rightarrow \infty$, is related to the Hausdorff dimension of Lagrangian regular points for the inviscid Burgers equation with FBM initial velocity. We produce computational evidence in favor of a power asymptotics for $p_{T}$. The data do not reject the hypothesis that the exponent $\theta$ of the power law is related to the similarity parameter $H$ of fractional Brownian motion as follows: $\theta=-(1-H)$ for the interval $(-\mathrm{T}, \mathrm{T})$ and $\theta=-H(1-H)$ for $(0, T)$. The point 0 is special in that IFBM and its derivative both vanish there.
\end{abstract}

Key words: Fractional Brownian motion, Burgers equation, fractality, large excursions

* Observatoire de la Cote d'Azur, CNRS UMR 6529, BPU 229, 06304, Nice Cedex 4, France, molchan@mitp.ru

$\dagger$ International Institute of Earthquake Prediction Theory and Mathematical Geophysics 79, b2, Warshavskoe shosse 113556 Moscow, Russia. khokhlov@mitp.ru, khokhlov@ipgp.jussieu.fr 


\section{Introduction}

Sinai [18] and Frisch and associates [17] initiated in 1992 the study of fractal and multifractal properties of solutions of the inviscid Burgers equation with initial velocity $u_{0}(x)$ specified by a self-similar random process. That last circumstance guarantees that the solution is self-similar in the large. In particular, one could be interested in finding the Hausdorff dimension of the set of regular Lagrangian points $\mathrm{S}$ that describe the initial locations of those fluid particles which have not collided until a fixed time $t_{0}$. The original model of $u_{0}(x)$ was fractional Brownian motion $(\mathrm{FBM}), b_{H}(x)$, with similarity parameter $0<H<1$.

By now the Sinai-Frisch program has been carried out for special Markovian models of $u_{0}(x)$ alone: Sinai [18] has found the dimension $S$ for Brownian motion case, i.e., $u_{0}(x)=b_{1 / 2}(x)$; Bertoin [3] discovered for this case that the solution $u\left(t=t_{0}, x\right)$ admits of an exact probabilistic description in terms of a stable Levy process. One can then find a multifractal description of the solution $x \rightarrow u\left(t_{0}, x\right)$ (the relevant references are $[6,7,8]$ ). Additionally, Bertoin [3] found that the Hausdorff dimension of Lagrangian regular points is $h$, if $u_{0}(x)$ is a stable Lévy process of index $\alpha=h^{-1} \in(1,2]$ with no positive jumps (see also [20]).

The nonmarkovian case $u_{0}(x)=b_{H}(x), H \neq 1 / 2$ has proved extremely difficult for analysis. Handa [5] found simple arguments to derive a lower bound on the dimension of $S$, namely, $\operatorname{dim} S \geq H$. The exact equality $\operatorname{dim} S=H$ is known as a hypothesis $[17,19]$ since 1992. Among methods developed for analyzing the nonmarkovian case $u_{0}(x)$, the Sinai approach is of particular interest. For the case $u_{0}(x)=b_{1 / 2}(x)$ this method [18] relates the estimation of the dimension of $S$ to the asymptotic behavior of the probability

$$
p_{T}=P\left\{\xi(x)<1, \quad x \in \Delta_{T}\right\}
$$

for integral Brownian motion

$$
\xi(x)=\int_{0}^{x} b_{1 / 2}(s) d s \quad \text { and } \quad \Delta_{T}=(0, T), \quad T \gg 1 .
$$

As a matter of fact (see below), one has to deal with a problem that is rather popular in physical and technical applications: find the probability of a large excursion for a random process $\eta(x)$, i.e., $P\{\eta(x)>0,1<x<T\}, T \gg 1$. A review of the problem can be found in [14]. Sinai has shown that the quantity $p_{T} \cdot T^{1 / 4}$ is bounded away from 0 and $\infty$ as $T \rightarrow \infty$ under the conditions (2). That estimate was repeatedly refined and generalized [9, 10, 11].

We show below that the upper bound $\operatorname{dim} S \leq H$ under the conditions $u_{0}(x)=b_{H}(x)$ follows from an estimate of $p_{T}$ for the integral of fractional Brownian motion (IFBM): $\xi(x)=\int_{0}^{x} b_{H}(s) d s$ when considered in the bilaterally expanding interval $\Delta_{T}=(-T, T)$. 
The work [15] clarifies the asymptotic problem of $p_{T}$ for intervals $(0, T)$ and $(-T, T)$ in the case of fractional Brownian motion: $\xi(x)=b_{H}(x)$. It transpires that in this case

$$
\ln p_{T}=-(1-H) \ln T(1+o(1)), \quad \Delta_{T}=(0, T) .
$$

On the other hand, when $\Delta_{T}=\{x:|x|<T\}$, the leading term in the log asymptotics of $p_{T}$ is independent of $H$. More generally, suppose $b_{H}(x), x \in R^{d}$ is FBM with multidimensional time; in that case

$$
\ln p_{T}=-d \ln T(1+o(1)), \quad \Delta_{T}=\left\{x \in R^{d}:|x|<T\right\} .
$$

The last asymptotics is due to the fact that the probability density for the position of the maximum of FBM exists in the sphere $\{|x|<1\}$. A generalization of this fact is given below.

We present theoretical and computational evidence in favor of the following asymptotics for IFBM:

$$
\ln p_{T}= \begin{cases}-(1-H) \ln T(1+o(1)), & \Delta_{T}=(-T, T) \\ -H(1-H) \ln T(1+o(1)), & \Delta_{T}=(0, T) .\end{cases}
$$

The first of these asymptotic expressions corroborates the hypothesis $\operatorname{dim} S=$ $H$, so is not unexpected, while the second is, considering that the exponent $\theta(H)=H(1-H)$ has the point of symmetry $H=1 / 2$.

Because IFBM is a self-similar process, the distribution of its maximum in $\Delta=(0,1)$ or $(-1,1), F_{\max }(x)$, is related to $p_{T}$ through $p_{T}=F_{\max }\left(T^{-(1+H)}\right)$. Importantly, our calculation was performed for a series of statistics: the maximum $M=\max$ IFBM; the position of the maximum $M,|G|$; the occupation

time $A^{+}=\int_{\Delta} \mathbf{1}_{\xi(x)>0} d x$ of IFBM above zero; and the rightmost zero of IFBM in $(0, T), Z$. The distributions of these statistics (one should use $F_{\max }\left(x^{1+H}\right)$ when $M$ is considered) have identical asymptotics as $x \rightarrow 0$, but depend on interval type: $\Delta=(0,1)$ or $(-1,1)$. When $\Delta=(-1,1)$, they provide independent evidence in favor of the hypothesis $\operatorname{dim} S=H$.

The rest of this paper is organized as follows. Section 2 reduces the evaluation of $\operatorname{dim} S \leq H$ to the asymptotic distributions of $M, G, A^{+}$and $Z$ near zero. Section 3 discusses the modeling of IFBM, while section 4 presents numerical evaluations of the distributions listed above and some theoretical arguments to support our conclusions.

\section{Regular Lagrangian points and the nonexceedance of level}

We now define more exactly the notions used in Introduction. We consider the Burgers equation 


$$
\partial_{t} u+u \partial_{x} u=\nu u_{x x}, \quad \nu \downarrow 0,
$$

with continuous initial conditions $u(0, x)=u_{0}(x)$ and the velocity potential $U(x)=\int_{0}^{x} u_{0}(x) d x=o\left(x^{2}\right), x \rightarrow \infty$. The solution at $t_{0}=1$ has the form $u(x)=x-a(x)$, where $a(x)$ can be found from $U(x)$ as follows. Construct a convex minorant $C(x)$ for $U(x)+x^{2} / 2$. In that case its derivative $C^{\prime}(x)$ is nondecreasing and has finite limits from the left and from the right. We now complete the definition of $C^{\prime}(x)$ in continuity on the right. In that case, according to Hopf (see, e.g., $[18,21]), a(x)$ is identical with the inverse function of $C^{\prime}(x)$. The set of points where $C^{\prime}(x)$ is increasing, i.e., the topological support of the measure $d C^{\prime}(x)$ or the closure of the set $\{a(x), x \in R\}$, defines the set $S$ of regular Langrangian points in the Burgers problem. The dynamics of completely inelastic particles on $R^{1}$ can be related to the Burgers equation: each infinitesimal particle located at $x$ has a mass $d x$ and an initial momentum $d U(x)$. On colliding the particles coalesce and continue movement following the conservation laws of mass and momentum. Particles that have not collided until time $t_{0}=1$ make up the set $S$ in the Lagrangian coordinates. The initial conditions $u_{0}(x)$ will be considered to be fractional Brownian motion $b_{H}(x)$, i.e., a Gaussian process with zero mean and structural function $E\left|b_{H}(x)-b_{H}(y)\right|^{2}=|x-y|^{2 H}$ where $0<H<1$. In virtue of the Kolmogorov theorem the paths of $b_{H}(x)$ can be treated as continuous a.s. The process $b_{H}(x)$ is self-similar, i.e., $b_{H}(\Lambda x) \stackrel{\mathrm{d}}{=} \Lambda^{H} b_{H}(x)$, where $\stackrel{\mathrm{d}}{=}$ denotes equality of finite-dimensional distributions.

Theorem 1. 1. The set of regular Lagrangian points $S$ in the Burgers problem (3) with $u_{0}(x)=b_{H}(x)$ has a.s. dimension $H$, if for any $\varepsilon>0$ and $T \rightarrow \infty$ one of the following requirements is fulfilled:

$$
\begin{aligned}
& P\left\{y(x):=\int_{0}^{x} b_{H}(s) d s<1, x \in \Delta_{T}\right\}<T^{-(1-H)+\varepsilon,} \\
& P\left(y(x)<0, x \in \Delta_{T},|x|>1\right)<T^{-(1-H)+\varepsilon}, \\
& P\left(\left|G\left(\Delta_{T}\right)\right|<1\right)<T^{-(1-H)+\varepsilon}, \\
& P\left\{\int_{\Delta_{T}} \mathbf{1}_{y(x)>0} d x<1,\left|G\left(\Delta_{T}\right)\right|<T\right\}<T^{-(1-H)+\varepsilon},
\end{aligned}
$$

where $\Delta_{T}=(-T, T), G\left(\Delta_{T}\right)$ is the position of the maximum of $y(x)$ in $\Delta_{T}$.

2. If one of type $A-D$ probabilities $p_{T}$ has an asymptotics of the form $\log p_{T}=-\theta \log T(1+o(1))$, the probabilities of the other types have the same asymptotics. This statement also holds for $\Delta_{T}=(0, T)$ with the probability $P\left(Z_{T}<1\right)$ in addition to $(A-D)$, where $Z_{T}$ is the rightmost zero of $y(x)$ in $(0, T)$.

The proof of the theorem will be preceded by two lemmas.

Lemma 1. $\operatorname{dim} S \leq H$, if for any $\varepsilon>0$ there exists a $\delta_{0}=\delta_{0}(\varepsilon)$ such that one has for arbitrary $x \in R^{1}$ : 


$$
P(S \cap B(x, \delta) \neq \phi)<\delta^{(1-H)-\varepsilon}, \delta<\delta_{0}
$$

where $B(x, \delta)$ is a ball of radius $\delta$ centered at $x$.

Proof. Cover the interval $\Delta=[a, b]$ with intervals $B_{i}(\delta)$ of length $\delta$ with overlappings of length $\delta / 2$. Consider the measure $\mu(d x)=d C^{\prime}(x)$ with support $S$. The elements $\tilde{B}_{i}$ in $\left\{B_{i}(\delta)\right\}$ for which $\mu\left(B_{i}\right)>0$ will then form a cover $S \cap \Delta$. In view of (4)

$$
\begin{gathered}
E \sum\left|\tilde{B}_{i}(\delta)\right|^{H+2 \varepsilon}=E \sum\left|B_{i}(\delta)\right|^{H+2 \varepsilon} \mathbf{1}_{\mu\left(B_{i}\right)>0} \\
<\delta^{H+2 \varepsilon} \cdot 2|\Delta| \delta^{-1} \cdot \delta^{(1-H)-\varepsilon}=c \delta^{\varepsilon}
\end{gathered}
$$

where $|\Delta|$ is the length of $\Delta$. By Chebyshev's inequality

$$
P\left(\sum\left|\tilde{B}_{i}\right|^{H+2 \varepsilon}>a\right)<c \delta^{\varepsilon} / a .
$$

Consider a sequence $\delta_{n}$ such that $\sum \delta_{n}^{\varepsilon}<\infty$. The Borel-Cantelli lemma then yields

$$
\sum\left|\tilde{B}_{i}\left(\delta_{n}\right)\right|^{H+2 \varepsilon}<a, \quad n>n(\omega)
$$

Since $a$ is arbitrary:

$$
\lim \sup _{n} \sum\left|\tilde{B}_{i}\left(\delta_{n}\right)\right|^{H+2 \varepsilon}=0 \quad \text { a.s. }
$$

However, in that case one has $\operatorname{dim}(S \cap \Delta) \leq H+2 \varepsilon$. Since $\varepsilon>0$ and $\Delta$ are arbitrary, one has $\operatorname{dim} S \leq H$.

Lemma 2. The conditions of Lemma 1 are fulfilled, if

$$
P\left\{\int_{0}^{x} b_{H}(s) d s<1, \quad|x|<T\right\}<T^{-(1-H)+\varepsilon}, \quad \forall \varepsilon>0 .
$$

as $T \rightarrow \infty$.

Proof. The process $y(x)=\int_{0}^{x} b_{H}(s) d s+x^{2} / 2$ can be represented in the form

$$
\begin{aligned}
y(x) & =\int_{0}^{c}\left(b_{H}(s)+s\right) d s+\left(b_{H}(c)+c\right)(x-c)+ \\
& +\int_{c}^{x}\left[b_{H}(s)-b_{H}(c)+(s-c)\right] d s=L\left(x^{\prime}\right)+\int_{0}^{x^{\prime}}\left(\tilde{b}_{H}(s)+s\right) d s,
\end{aligned}
$$


where $L\left(x^{\prime}\right)$ is a linear function of $x^{\prime}=x-c$, and $\tilde{b}_{H}(x)=b_{H}(c+x)-b_{H}(c) \stackrel{\mathrm{d}}{=}$ $b_{H}(x)$. The convex minorants of $y$ and $\tilde{y}=\int_{0}^{x^{\prime}}\left(\tilde{b}_{H}(s)+s\right) d s$ differ by the linear function $L\left(x^{\prime}\right)$. Hence the fractal properties of the measure $\mu(d x)=d C^{\prime}(x)$ are invariant under translation along the $x$-axis (this observation is due to $\mathrm{U}$. Frisch). Consequently, it is sufficient to prove (4) for $S^{\prime}=S \cap(-\delta / 2, \delta / 2)$.

Let $\Delta=(-\delta / 2, \delta / 2)$ contain a point of growth $x_{0}$ for the measure $d \mu$. That means that the curve $f(x)=U(x)+x^{2} / 2$ and its convex minorant $C(x)$ do not lie below the tangent of $f(x)$ at the point $x_{0}$, and $C\left(x_{0}\right)=f\left(x_{0}\right)$. The event $\left\{x_{0} \in \Delta\right\}$, to be called $A$ here, can be written as

$$
\begin{aligned}
A & =\left\{\exists x_{0}:\left|x_{0}\right|<\delta / 2 ; \int_{0}^{x}\left(b_{H}(s)+s\right) d s\right. \\
& \left.>\int_{0}^{x_{0}}\left(b_{H}(s)+s\right) d s+\left(b_{H}\left(x_{0}\right)+x_{0}\right)\left(x-x_{0}\right), \forall x \in R^{1}\right\} .
\end{aligned}
$$

Let us modify event $A$ to become $A_{1}$, i.e., we assume that the equality in the formulation of $A$ is true for $|x|<1$ only. To emphasize the fact that $A_{1}$ depends on the process $b_{H}(x)+x=\xi(x)$, we will write $A_{1}=A_{1}[\xi]$.

One has

$$
P(A) \leq P\left(A_{1}\right)=E \mathbf{1}_{A_{1}\left[b_{H}+\varphi\right]}=E \mathbf{1}_{A_{1}\left[\tilde{b}_{H}\right]} \pi\left(\tilde{b}_{H}\right)
$$

where $\varphi(x)=x$ and $\pi$ is the Radon-Nikodim derivative of two Gaussian measures corresponding to the processes $\tilde{b}_{H}-\varphi$ and $\tilde{b}_{H}$ in $[-1,1]$. Note that $\tilde{b}_{H}$ is an FBM process. The function $\varphi$ is smooth and vanishes at zero. For this reason the above measures are mutually absolutely continuous [16]. By the CameronMartin relation $\ln \pi\left(\tilde{b}_{H}\right)$ is a Gaussian variable with mean $-c_{H}^{2} / 2$ and variance $c_{H}^{2}$, where $c_{H}=\|\varphi\|$ and $\|\cdot\|$ is the norm in Hilbert space $H_{B}$ of functions on $\Delta=[-1,1]$ with reproducing kernel $B(x, y)=E b_{H}(x) b_{H}(y)$. The constant $c_{H}$ is finite and can be found in explicit form as indicated by Molchan and Golosov $[16]$.

Applying Hölder's inequality to the right-hand side of (5), one gets

$$
P(A)<P\left(A_{1}\left[\tilde{b}_{H}\right]\right)^{1-\varepsilon}\left(E \pi^{1 / \varepsilon}\right)^{\varepsilon}=P\left(A_{1}\left[\tilde{b}_{H}\right]\right)^{1-\varepsilon} c_{\varepsilon}
$$

where $c_{\varepsilon}=\exp \left(\frac{1}{2}\left(\varepsilon^{-1}-1\right) c_{H}^{2}\right)$.

We now evaluate $P\left(A_{1}\left[b_{H}\right]\right)$. One has

$$
\begin{aligned}
P\left(A_{1}\left[b_{H}\right]\right) & =P\left\{\exists x_{0}:\left|x_{0}\right|<\delta / 2\right. \\
\int_{0}^{x} b_{H}(s) d s & \left.>\int_{0}^{x_{0}} b_{H}(s) d s+b_{H}\left(x_{0}\right)\left(x-x_{0}\right),|x|<1\right\} \\
& =P\left\{\exists x_{0}:\left|x_{0}\right|<1 / 2 ; \int_{0}^{x} b_{H}(s) d s>a\left(x_{0}\right)+b\left(x_{0}\right) x,|x|<T\right\}
\end{aligned}
$$


where

$$
\begin{aligned}
T=\delta^{-1}, \quad\left|a\left(x_{0}\right)\right| & =\left|\int_{0}^{x_{0}} b_{H}(s) d s-b_{H}\left(x_{0}\right) x_{0}\right|<2 M, \\
\left|b\left(x_{0}\right)\right| & =\left|b_{H}\left(x_{0}\right)\right|<M=\max _{|x|<1 / 2}\left|b_{H}(x)\right| .
\end{aligned}
$$

We will use the Fernique inequality [4]

$$
P\left(M>\bar{c}_{H} u\right)<\exp \left(-u^{2} / 2\right)=T^{-a}, \quad u>u_{0},
$$

where $u=u_{T}=\sqrt{2 a \ln T}, c_{H}$ being a constant; the value of $a$ will be chosen later on. From this it follows that

$$
\begin{aligned}
P\left(A_{1}\left[b_{H}\right]\right) & <P\left\{A_{1}\left[b_{H}\right], \quad M<\bar{c}_{H} u_{T}\right\}+T^{-a} \\
& <P\left\{\int_{0}^{x} b_{H}(s) d s>-2 \bar{c}_{H} u_{T}-\bar{c}_{H} u_{T}|x|,|x|<T\right\}+T^{-a} \\
& =P\left\{\int_{0}^{x} b_{H}(s) d s<u_{T} \bar{c}_{H}(2+|x|),|x| \leq T\right\}+T^{-a} \\
& =P\left\{\int_{0}^{x} b_{H}(s) d s<4 \lambda_{T}^{-1}+2|x|,|x|<T^{\prime}\right\}+T^{-a},
\end{aligned}
$$

where $T^{\prime}=T / \lambda_{T}, \quad u_{T} \bar{c}_{H}=2 \lambda_{T}^{H}, \lambda_{T}=$ const $\cdot(\ln T)^{1 / 2 H}$. Here we have used the fact that $b_{H}(x)$ is a self-similar process and modified the interval $|x| \leq T$ to become $|x|<T^{\prime}$.

Define the function

$$
\varphi_{1}(x)=2 x \mathbf{1}_{|x|<1}+2 \operatorname{sgn}(x) \mathbf{1}_{|x|>1}=\frac{2}{\pi i} \int\left[e^{i x \lambda}-1\right] \frac{\sin \lambda}{\lambda^{2}} d \lambda .
$$

In that case (7) can be continued to get

$$
P\left(A_{1}\left[b_{H}\right]\right) \leq P\left\{\int_{0}^{x}\left(b_{H}(s)-\varphi_{1}(s)\right) d s<F(x),|x|<T^{\prime}\right\}+T^{-a},
$$

where

$$
F(x)= \begin{cases}-x^{2}+2|x|+4 \lambda_{T}^{-1}, & |x|<1 \\ 1+4 \lambda_{T}^{-1}, & |x|>1\end{cases}
$$

When $T$ is large, one has $F(x)<2$. For this reason the last estimate will merely become less precise, when $F$ is replaced with $F(x)=2$. The right-hand side 
of (9) can be evaluated by repeating the steps that have led to $(5,6)$. The substitution of $\tilde{b}_{H}$ for $b_{H}-\varphi_{1}$ combined with Hölder's inequality yield

$$
P\left(A_{1}\left[b_{H}\right]\right)<P\left\{\int_{0}^{x} b_{H}(s) d s<2,|x|<T^{\prime}\right\}^{1-\varepsilon} c_{\varepsilon}^{1}+T^{-a},
$$

where $c_{\varepsilon}^{1}=\exp \left(\left(\varepsilon^{-1}-1\right) b_{H}^{2} / 2\right), b_{H}^{2}=\left\|\varphi_{1}\right\|_{T}^{2} \leq\left\|\varphi_{1}\right\|_{\infty}^{2}$. Here $\|\cdot\|_{T}$ is the norm on $H_{B}$ for the interval $(-T, T)$. The spectral representations of the kernel

$$
B(t, s)=E b_{H}(t) b_{H}(s)=k_{H}^{-1} \int\left(e^{i x \lambda}-1\right)\left(e^{-i x \lambda}-1\right)|\lambda|^{-1-2 H} d \lambda
$$

and $\varphi_{1}($ see $(8))$ yield

$$
\left\|\varphi_{1}\right\|_{\infty}^{2}=k_{H} \int\left|\frac{2 \sin \lambda}{\pi \lambda^{2}}\right|^{2}|\lambda|^{1+2 H} d \lambda<\infty
$$

where $k_{H}=\int\left|e^{i \lambda}-1\right|^{2}|\lambda|^{-1-2 H} d \lambda$.

The final result is

$$
P(A) \leq P\left(A_{1}\left[b_{H}\right]\right)^{1-\varepsilon} c_{\varepsilon}<\left(p_{T^{\prime}}^{1-\varepsilon} c_{\varepsilon}^{1}+T^{-a}\right)^{1-\varepsilon} c_{\varepsilon},
$$

where $p_{T^{\prime}}=P\left\{\int_{0}^{x} b_{H}(s) d s<2,|x|<T^{\prime}\right\}$.

Let $p_{T}<T^{-(1-H)+\varepsilon_{1}}$ for large $T$. Take $a>1-H$ and choose $\varepsilon$ from the requirement $c_{\varepsilon}^{1} \cdot c_{\varepsilon}=T^{\prime \varepsilon_{1}}$, i.e., $\varepsilon=c \varepsilon_{1}^{-1} / \ln T^{\prime}$. Inequality (10) can then be continued:

$$
P(A)<c_{1} T^{\prime-(1-H)+2 \varepsilon_{1}},
$$

where $c_{1}=\exp \left(2(1-H) c \varepsilon_{1}^{-1}\right)$. Recalling that $T^{\prime}=T(\ln T)^{-1 / 2 H} \cdot c_{2}$, one obtains the desired estimate $P(A)<T^{-(1-H)+3 \varepsilon_{1}}, T>T_{0}\left(\varepsilon_{1}\right), T=\delta^{-1}$.

Proof of Theorem 1. The inequality $\operatorname{dim} S \geq H$ was derived by Handa [5]. The opposite inequality $\operatorname{dim} S \leq H$ follows from Lemmas 1 and 2 and condition $(A)$ of Theorem 1 . To prove the theorem under condition $(B)$, we note that the event $\left\{\int_{0}^{x} b_{H}(s) d s<c,|x|<T\right\}$ can be represented as

$$
\left\{\int_{0}^{x}\left(b_{H}(s)-\varphi(s)\right) d s<\psi(x), \quad|x|<T\right\},
$$

where $\varphi, \psi$ are smooth finite functions: $\psi \equiv 0$ when $|x| \geq 1$ and $\psi>0$ when $|x|<1$, while $\varphi=0$ when $|x|<1 / 2$ and $|x|>1$. Repeating the translation procedure for the samples: $b_{H}(s)-\varphi(s) \rightarrow \tilde{b}_{H}(s)$ and using Hölder's inequality, we get 


$$
\begin{aligned}
p_{T}: & =P\left\{\int_{0}^{x} b_{H}(s) d s<1,|x|<T\right\} \\
& <c_{\varepsilon} P\left\{\int_{0}^{x} b_{H}(s) d s<\psi(x),|x|<T\right\}^{1-\varepsilon} \\
& <c_{\varepsilon} P\left\{\int_{0}^{x} b_{H}(s) d s<0,1<|x|<T\right\}^{1-\varepsilon},
\end{aligned}
$$

where $c_{\varepsilon}=\exp \left(\frac{1}{2} \varepsilon^{-1} \cdot c_{\varphi}^{2}\right), c_{\varphi}<k_{H} \int|\hat{\varphi}(\lambda)|^{2}|\lambda|^{1+2 H}$ and $\hat{\varphi}$ is the Fourier transform of $\varphi$. One has $c_{\varphi}<\infty$, because $\varphi$ is smooth and finite. Choose $\varepsilon=\varepsilon_{T}$ from the requirement $c_{\varepsilon}=\mathcal{L}_{T}$, where $\mathcal{L}_{T}$ is a slowly varying function. Take $\mathcal{L}_{T}=\ln T$, say, then $\varepsilon_{T}^{-1}=c \ln \ln T$. The result is

$$
p_{T}<\mathcal{L}_{T} P\left(\int_{0}^{x} b_{H}(s) d s<0,1<|x|<T\right)^{1-\varepsilon_{T}} .
$$

When $(B)$ holds, one has

$$
p_{T}<\left(T^{-(1-H)+\varepsilon_{1}}\right)^{1-\varepsilon_{T}} \mathcal{L}_{T}<T^{-(1-H)+\varepsilon_{2}}, T \gg 1,
$$

i.e., the implication $(B) \rightarrow(A)$ is true.

The inequality

$$
P\left(\int_{0}^{x} b_{H}(s) d s<0,1<|x|<T\right)<P(|G(-T, T)|<1),
$$

where $G(\Delta)$ is the position of the maximum of IFBM in $\Delta$, yields the implication $(C) \rightarrow(B)$. Lastly, under $(B)$ the position of the maximum of IFBM is $|G|<1$, while the occupation time of IFBM above 0 is below 2 . Hence $(D) \rightarrow(B)$.

Let us prove $(A) \rightarrow(C)$. Below, $G_{T}$ is the position of the maximum of IFBM in $(-T, T)$ and $M_{a}=\max _{|x|<a} \operatorname{IFBM}$. One has

$$
\left.P\left(\left|G_{T}\right|<1\right)<P\left(\left|G_{T}\right|<1\right), M_{1}<c_{T}\right)+P\left(M_{1}>c_{T}\right) .
$$

If $c_{T}=\sqrt{2 a \ln T}$, then the Fernique estimate [4] yields

$$
P\left(M_{1}>c_{T}\right)<c T^{-a^{\prime}}, T>T_{0},
$$

where $a^{\prime}=a / \sigma^{2}$ and $\sigma^{2}=E|\operatorname{IFBM}(1)|^{2}=(2 H+2)^{-1}$. Also,

$$
P\left(\left|G_{T}\right|<1, M_{1}<c_{T}\right)<P\left(M_{T}<c_{T}\right) .
$$


Since IFBM is self-similar, one has $M_{T} \stackrel{\mathrm{d}}{=} \lambda^{1+H} M_{T^{\prime}}$, when $T=\lambda T^{\prime}$. Take $\lambda$ from the requirement $\lambda^{1+H}=c_{T}$. Then $P\left(M_{T}<c_{T}\right)=P\left(M_{T^{\prime}}<1\right)$.

To sum up,

$$
P\left(\left|G_{T}\right|<1\right)<P\left(M_{T^{\prime}}<1\right)+o\left(T^{-a}\right)
$$

where $T^{\prime}=c T(\ln T)^{-\rho}, \rho=(1-H)^{-1}$, while the parameter $a>0$ is arbitrary. When $a>(1-H)$, the implication $(\mathrm{A}) \rightarrow(\mathrm{C})$ is obvious.

We are going to prove $(\mathrm{A}) \rightarrow(\mathrm{D})$. Let $A_{T}^{+}$be the occupation time of $y(x)=$ $\operatorname{IFBM}(x)$ above zero in $\Delta_{T}=(-T, T)$. One has

$$
P\left(A_{T}^{+}<1,\left|G_{T}\right|<T\right) \leq P\left(M_{T}<c_{T}\right)+P\left(M_{T}>c_{T}, A_{T}^{+}<1,\left|G_{T}\right|<T\right),
$$

where $c_{T}$ will be specified below.

Let $\Delta_{T}=\cup \Delta_{k}, \Delta_{k}=(k, k+1)$ and $M_{k}=\max \left\{y(x), x \in \Delta_{k}\right\}$. If the event $\mathcal{B}=\left\{M_{T}>c_{T}, A_{T}^{+}<1,\left|G_{T}\right|<T\right\}$ occurs, one will have the following for the interval $\Delta_{k}$ which contains $G_{T}: M_{k}>c_{T}, y(x)$ and $y^{\prime}(x)=b_{H}(x)$ have zeroes in $\Delta_{k}$. Indeed, if $y(x) \neq 0$, then $y(x)>0$ in $\Delta_{k}$ and $A_{T}^{+} \geq 1$. Consequently,

$$
P(\mathcal{B})<\sum_{k} P\left\{\max \left(\left(y\left(x_{1}\right)-y\left(x_{2}\right)\right)>c_{T}, x_{1}, x_{2} \in \Delta_{k}\right), S_{k}\right\}:=\sum_{k} p_{k},
$$

where $S_{k}$ means that $b_{H}(x)$ has a zero in $\Delta_{k}$.

We are going to evaluate $p_{k}$ :

$$
\begin{aligned}
p_{k} & <P\left\{\max \left[\left(y\left(x_{1}\right)-y\left(x_{2}\right)\right), x_{1}, x_{2} \in \Delta_{k}\right]>c_{T},\left|b_{H}(k)\right|<c_{T} / 2\right\} \\
& +P\left\{\left|b_{H}(k)\right|>c_{T} / 2, S_{k}\right\}:=p_{k, 1}+p_{k, 2} .
\end{aligned}
$$

One has

$$
\begin{aligned}
p_{k, 2} & <P\left\{\max \left(\left|b_{H}\left(x_{1}\right)-b_{H}\left(x_{2}\right)\right|, x_{1}, x_{2} \in \Delta_{k}\right)>c_{T} / 2\right\} \\
& =P\left\{\max \left(\left|b_{H}\left(x_{1}\right)-b_{H}\left(x_{2}\right)\right|, x_{1}, x_{2} \in \Delta_{0}\right)>c_{T} / 2\right\}
\end{aligned}
$$

Here we have used the fact that $b_{H}(x)$ has stationary increments. In virtue of the Fernique inequality [4]

$$
p_{k, 2}<c \exp \left(-\frac{1}{2}\left(c_{T} / c_{b}\right)^{2}\right)
$$

where $c$ is an absolute constant, while $c_{b}$ is a function of $H$.

One proceeds in a similar manner to evaluate $p_{k, 1}$ : 


$$
y\left(x_{1}\right)-y\left(x_{2}\right)=\int_{x_{2}}^{x_{1}}\left(b_{H}(s)-b_{H}(k)\right) d x+b_{H}(k)\left(x_{1}-x_{2}\right) .
$$

If $\max \left[y\left(x_{1}\right)-y\left(x_{2}\right)\right]>c_{T}$ in $\Delta_{k} \times \Delta_{k}$ and $\left|b_{H}(k)\right|<c_{T} / 2$, then

$$
\max \int_{x_{2}}^{x_{1}}\left[b_{H}(s)-b_{H}(k)\right] d s>c_{T} / 2 .
$$

Consequently,

$$
\begin{aligned}
p_{k, 1} & <P\left\{\max \left[\int_{x_{2}}^{x_{1}}\left(b_{H}(s)-b_{H}(k)\right) d s, x_{1}, x_{2} \in \Delta_{k}\right]>c_{T} / 2\right\} \\
& =P\left\{\max \left[\int_{x_{1}}^{x_{2}} b_{H}(s) d s, x_{1}, x_{2} \in \Delta_{0}\right]>c_{T} / 2\right\} .
\end{aligned}
$$

Here again, we have used the relation $b_{H}(x)-b_{H}(k) \stackrel{\mathrm{d}}{=} b_{H}(x-k)$ with a fixed $k$. The use of the Fernique inequality [4] yields

$$
p_{k, 1}<c \exp \left(-\frac{1}{2}\left(c_{T} / 2 c_{y}\right)^{2}\right)
$$

where $c_{y}$ is a function of $H$. Combining the estimates of $p_{k, 1}$ and $p_{k, 2}$ and assuming $c_{T}=\max \left(c_{b}, 2 c_{y}\right) \sqrt{2 a \log T}$, one gets

$$
p_{k}=p_{k, 1}+p_{k, 2}<c T^{-a} .
$$

However, one then has $P(\mathcal{B})<2 c T^{-a+1}$ and

$$
\begin{aligned}
P\left(A_{T}^{+}<1,\left|G_{T}\right|<T\right) & \leq P\left(M_{T}<c_{T}\right)+O\left(T^{-a+1}\right) \\
& =P\left(M_{T^{\prime}}<1\right)+O\left(T^{-a+1}\right),
\end{aligned}
$$

where $T^{\prime}=c T(\log T)^{-\rho}, \rho=(1-H)^{-1}$. Hence $(A) \rightarrow(D)$.

Consider the second part of the theorem. Let $p_{T}(\Theta)$ be the probabilities that appear in the first part of the theorem, where $\Theta$ denotes the condition $A, B, C$ or $D$. It has been shown above that, when $T \gg 1$,

$$
\begin{aligned}
& p_{T}(A)<p_{T}(B)^{1-\varepsilon_{T}} \mathcal{L}_{T}, p_{T}(B)<p_{T}(C), \\
& p_{T}(C)<p_{T^{\prime}}(A)+O\left(T^{-a}\right), p_{T}(D)<p_{T^{\prime}}(A)+O\left(T^{-a+1}\right),
\end{aligned}
$$

where $a>0$ is any fixed number, $\mathcal{L}_{T}$ is a slowly varying function of $T, \varepsilon_{T}=o(1)$, $T \rightarrow \infty$ and $T^{\prime}=c T(\ln T)^{-\rho}, \rho=(1-H)^{-1}$. A trivial corollary of these is that 
all the $p_{T}(\Theta)$ have the asymptotics $\ln p_{T}(\Theta)=-\theta \ln T(1+o(1))$, provided the asymptotics is true for at least a single quantity of the type $\Theta=A, C$ or $D$.

Our proof has not relied significantly on the type of the interval $\Delta_{T}:(-T, T)$ or $(0, T)$. For this reason our conclusion that the asymptotics of $p_{T}(\Theta)$ are identical also holds for $(0, T)$.

We conclude by noting that, if $\Delta_{T}=(0, T)$, then $p_{T}(B)=P(y(x)>0,1<$ $t<T)$. Consequently, if $Z_{T}$ is the rightmost zero of $y(x)$ in $(0, T)$, then $P\left(Z_{T}<\right.$ 1) $=2 p_{T}(B)$.

\section{The generation of IFBM}

We are going to use Monte Carlo techniques in order to evaluate the probabilities $p_{T}(\Theta)$ with $\Theta=A, C, D$ in Theorem 1 for the process $y(x)=\int_{0}^{x} b_{H}(s) d s$ in the following intervals of $\Delta_{T}:(0, T)$ and $(-T, T)$. The probabilities in question are small, $p_{T} \rightarrow 0$ as $T \uparrow \infty$, hence the IFBM generation should be exact for a discrete sequence $\left\{x_{k}, k=1, \ldots, T\right\}$. Since $y(x)$ is a self-similar process, it is sufficient to use integer points $x_{k}=k$. In that case $\{y(k / T)\} \stackrel{\mathrm{d}}{=}\left\{T^{-(1+H)} y(k)\right\}$, while the probabilities $p_{T}(\Theta)$ can obviously be expressed in terms of the statistics $M=\max _{\Delta_{1}} y(x), G=\arg \max _{\Delta_{1}} y(x), A^{+}=\int_{\Delta_{1}} \mathbf{1}_{y>0} d x$ of the process $\left\{y(x), x \in \Delta_{1}\right\}$, where $\Delta_{1}=(0,1)$ or $(-1,1)$, as follows:

$p_{T}(A)=F_{M}\left(T^{-(1-H)}\right) ; \quad p_{T}(C)=F_{|G|}\left(T^{-1}\right) ; \quad p_{T}(D)=\hat{F}_{A}\left(T^{-1}\right) F_{G}(1-0)$

where $F_{\xi}$ is the distribution of $\xi$ and $\hat{F}_{A}$ is the conditional distribution of $A^{+}$ given $|G| \neq 1$.

The generation of $\{y(k), k=0, \ldots, T\}$. The sequence $\{y(k), k=0, \ldots, T\}$ is Gaussian and has stationary second increments, i.e., the sequence

$$
\eta_{k}=y(k-1)-2 y(k)+y(k+1), \quad k=1, \ldots, T-1,
$$

has a Toeplitz correlation matrix $\left[\mu_{i-j}\right]$, where

$$
\mu_{k}=c_{q}\left[|k-2|^{q}-4|k-1|^{q}+6|k|^{q}-4|k+1|^{q}+|k+2|^{q}\right]
$$

and $c_{q}=[2 q(q-1)]^{-1}, q=2 H+2$.

The second differences (11) combined with the initial conditions $y(0)=0$ and $y(1)$ are sufficient to uniquely reconstruct the sequence $\{y(k), k=0, \ldots, T\}$. One can assign $y(1)$ by using the decomposion $y(1)=\hat{y}(1)+y^{\perp}(1)$ into the predictable part $\hat{y}(1)=E\left(y(1) \mid \eta_{1}, \ldots, \eta_{T-1}\right)$ of $y(1)$ and the part $y^{\perp}(1)$ that cannot be predicted from the data $\left.\left\{\eta_{i}, i=1, \ldots, T-1\right)\right\}$. In that case

$$
y(1)=\sum_{k=1}^{T-1} z_{k} \eta_{k}+\sigma \varepsilon_{0}
$$


Here, $\mathbf{z}=\left(z_{1}, \ldots, z_{T-1}\right)^{\prime}$ is the solution of the linear equation:

$$
\left[\mu_{i-j}\right]_{1}^{T-1} \mathbf{z}=\mathbf{m},
$$

where the vector $\mathbf{m}$ has the components

$$
m_{k}=E y(1) \eta_{k}=\boldsymbol{\Delta}\left[q|k|^{q-1}-|k|^{q}+|k-1|^{q}\right] c_{q}
$$

and $\boldsymbol{\Delta}$ is the difference operator of second order: $\boldsymbol{\Delta} f(k)=f(k-1)-2 f(k)+$ $f(k+1)$. The second term is $\sigma \varepsilon_{0}=y^{\perp}(1)$, where $\varepsilon_{0}$ is the standard Gaussian random variable which is independent of $\left\{\eta_{1}, \ldots, \eta_{T-1}\right\}$;

$$
\sigma^{2}=E\left[y^{\perp}(1)\right]^{2}=q^{-1}-\sum_{1 \leq k<T} z_{k} m_{k},
$$

because $q^{-1}=E|y(1)|^{2}$ and $E|\hat{y}(1)|^{2}=\sum_{1}^{T-1} z_{k} m_{k}$.

It thus appears that the exact generation of the sequence $\{y(k), k=0, \ldots, T\}$ reduces to the generation of the stationary Gaussian sequence $\left\{\eta_{k}, k=1, \ldots, T-\right.$ $1)\}$ with correlation function (12) and to the solution of the linear equation (14).

The generation of $\{y(k),|k|<T / 2\}$. For generating $y(x)$ in a bilateral interval, we note the following. Assume that $y(x)$ is IFBM in $(0, T)$, while $\tilde{y}(x)$ is IFBM in $\left(-T^{\prime}, T^{\prime \prime}\right), T^{\prime}+T^{\prime \prime}=T$. In that case

$$
\left\{y(x)-y\left(T^{\prime}\right)-y^{\prime}\left(T^{\prime}\right)\left(x-T^{\prime}\right), x \in(0, T)\right\} \stackrel{\mathrm{d}}{=}\left\{\tilde{y}\left(x-T^{\prime}\right), x \in(0, T)\right\}
$$

The left-hand side provides a key to how one is to transform the sequence $\{y(k), k=0, \ldots, T\}$ into an IFBM sequence that starts from the point $0<k_{0}<$ $T$. To do this one must also find the derivative $y^{\prime}\left(k_{0}\right)$. In a similar way as above:

$$
y^{\prime}\left(k_{0}\right)=E\left\{y^{\prime}\left(k_{0}\right) \mid \eta_{1}, \ldots, \eta_{T-1}\right\}+E\left\{y^{\prime}\left(k_{0}\right) \mid \varepsilon_{0}\right\}+y^{\prime \perp}\left(k_{0}\right)
$$

where the first two terms correspond to the predictable part of $y^{\prime}\left(k_{0}\right)$ based on the data $\left\{\eta_{1}, \ldots, \eta_{T-1}, \varepsilon_{0}\right\}$, while the third term corresponds to the unpredictable part of $y^{\prime}\left(k_{0}\right)$. The predictable part is

$$
E\left\{y^{\prime}\left(k_{0}\right) \mid \eta_{1}, \ldots, \eta_{T-1}, \varepsilon_{0}\right\}=\sum_{k=1}^{T-1} z_{k}^{\prime} \eta_{k}+a \varepsilon_{0},
$$

where $\left(z_{1}^{\prime}, \ldots, z_{T-1}^{\prime}\right)$ is the solution of $(14)$ with the right-hand side $\mathbf{m}^{\prime}=\left(m_{1}^{\prime}, \ldots, m_{T-1}^{\prime}\right)$. The components of $\mathbf{m}^{\prime}$ are

$$
m_{k}^{\prime}=E y^{\prime}\left(k_{0}\right) \eta_{k}=\boldsymbol{\Delta}\left[|k|^{q-1}+\left|k_{0}-k\right|^{q-1} \operatorname{sgn}\left(k_{0}-k\right)\right] q c_{q} .
$$


Hence

One has $a=E y^{\prime}\left(k_{0}\right) \varepsilon_{0}$. From (13) one derives $E y(1) y^{\prime}\left(k_{0}\right)=\sigma a+\sum_{k=1}^{T-1} z_{k} m_{k}^{\prime}$.

$$
\sigma a=c_{q} \cdot q\left[(q-1) k_{0}^{q-2}+1-k_{0}^{q-1}+\left(k_{0}-1\right)^{q-1}\right]-\sum_{k=1}^{T-1} z_{k} m_{k}^{\prime} .
$$

One has $y^{\prime \perp}\left(k_{0}\right)=\sigma^{\prime} \varepsilon^{\prime}$ where $\varepsilon^{\prime}$ is a standard Gaussian variable that is independent of $\left(\eta_{1}, \ldots, \eta_{n-1}, \varepsilon\right)$. The variance of the unpredictable part $\sigma^{\prime 2}$ can be found from the relation

$$
k_{0}^{2 H}=E\left[y^{\prime}\left(k_{0}\right)\right]^{2}=\sum_{k=1}^{T-1} z_{k}^{\prime} m_{k}^{\prime}+a^{2}+\sigma^{\prime 2} .
$$

To sum up, the exact generation of $\left\{y(k), k=0, \ldots, T ; y\left(k_{0}\right)=y^{\prime}\left(k_{0}\right)=0\right\}$ requires that an equation like (14) should be solved twice.

The generation of $\left\{\eta_{k}\right\}$. Bardet et al. [2] provide a review of the methods which allow generation of Gaussian stationary sequences with a prescribed correlation function. We use the progressive Schur algorithm [1], which is a Levinson-Durbin method. The Generalized Schur algorithm can be used in the framework of this method for fast solution of equations like (14) by the GohbergSemenkul formula [1]. The generation of $\left\{\eta_{k}, k=1, \ldots, T-1\right\}$ by this method requires $O\left(T^{2}\right)$ operations. The computation is organized so as to minimize the amount of calculation needed for generating $N$ IFBM samples; the computational complexity is a linear function of $N$ and the storage capacity is of order $O\left(T^{2}\right)$.

The parameters $T$ and $N$ are equally important in the problem discussed. However, it appears that the increase of $T$ would not be effective from a priori considerations. The argument is as follows. We are interested in the distributions of $M, G, A^{+}$near zero where they are expected to behave like $x^{\theta} \mathcal{L}(x)$, where $\mathcal{L}$ is a slowly varying function. Since the approximation to IFBM is discrete, these distributions contain a positive atom at zero of size $p_{0}(T)=P\left(y(k) \leq 0, k \in \Delta_{T}\right)$. The probability is doubled for the statistic $Z$ (the rightmost zero of $y(t), t \in(0, T)$ ).

Note that $p_{0}(T) \geq P\left(y(x),|x|>1, x \in \Delta_{T}\right)$. Therefore, if the bounds given by Theorem 1 are explicit, $p_{0}(T)$ should not decrease faster than $T^{-\theta} \mathcal{L}^{\prime}(T)$. The rate of decrease becomes very low, when $\theta<1$. The expected value is $\theta=1-H$ for the interval $(-T, T)$ and $\theta \leq 1 / 4$ for $(0, T)$ (see below).

Given the above situation, it only remains to increase $N$ alone. This will provide good accuracy for empirical estimates of the distributions of $M, G, A^{+}$, $Z$ for discrete time. The discrete-time distributions cannot converge to the basic continuous ones faster that $T^{-\theta(H)}$ that we have at $x=0$.

\section{Evaluation of $\theta(H)$ and related results}


The bilateral IFBM process.

Figure 1 presents estimates of the distribution of $\left|G_{1 / 2}\right|\left(G_{a}\right.$ is the position of the maximum of $y(x)=\int_{0}^{x} b_{H}(s) d s$ in the interval $\left.(-a, a)\right)$. The distributions are given for $H: 0.1-0.9$ at increments of 0.1 . The estimates are based on $N=50,000$ samples of $y(x)$ with sample size $T=8194$. We use $\left|G_{1 / 2}\right|$ to demonstrate the asymptotics of $p_{T}$ from Theorem 1, because in this case the distribution discontinuity at $x=0$ bends the graph of the distribution on a $\log$ - $\log$ scale near zero to a lesser extent. We recall that the discontinuity of size $p_{0}(T)$ occurs for all the statistics $M, G$ and $A^{+}$, and is due to the presence of negative excursions in $y(x)$ on a discrete grid of $x$. The curves in Fig. 1 are well consistent with the asymptotics of type $F_{G}(x)=x^{\theta} \mathcal{L}(x)$, where $\mathcal{L}$ is a slowly varying function and $\theta=1-H$. Assuming $\mathcal{L}(x)=$ constant in $\left(x_{-}, x_{+}\right)$, one can construct the estimate of maximum likelihood $\hat{\theta}$ for $\theta$.

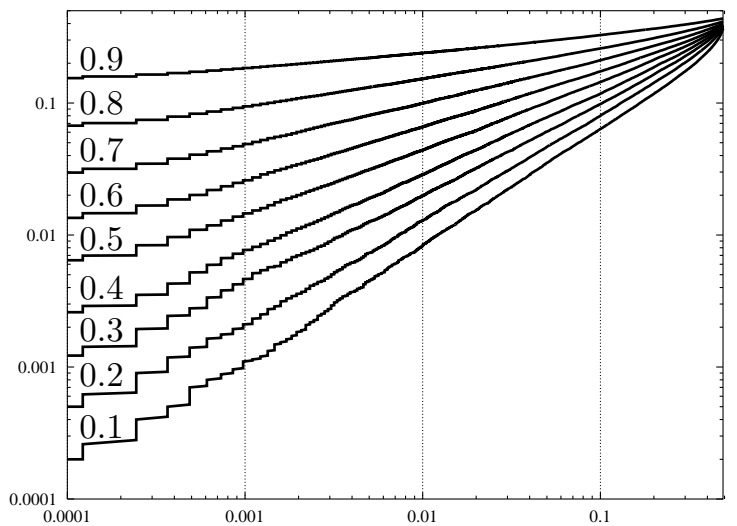

Figure 1: Distributions of $|G|$ for the position of maximum $G$ of the IFBM process in interval $\left(-\frac{1}{2}, \frac{1}{2}\right)$. Various values of $H$ are shown at the left hand part of each curve.

The estimate $\hat{\theta}$ was computed in intervals of the form $\left(10^{-3}, 10^{-2}\right) \times i, i=$ $1-5$. The choice of the initial point 0.001 is related to the fact that the sampling interval over time is $\Delta=T^{-1}=0.00012$, so that all curves in $(\Delta, 10 \Delta)$ slightly change their slopes due to the discontinuity of discrete-time distributions at zero. The deviation of $\hat{\theta}$ from the hypothetical $\theta_{0}=1-H$ does not exceed 0.03 , i.e., is less than $6 \%$ when $H \leq 0.5$. The error is large $(\geq 10 \%)$ for $0.6 \leq H \leq 0.9$ because of smallness of $\theta_{0}$. For $H \geq 0.6$ we have a slow convergence of the discrete-time distributions with $\Delta=T^{-1} \downarrow 0$ and difficulties in the choice of small $x$ to estimate $\theta$.

The argument to be given below provides a partial explanation of the nature of the asymptotics

$$
P\left(\left|G_{T}\right|<1\right)=T^{-(1-H)} \mathcal{L}_{T} .
$$

The function $y(x)$ is differentiable, hence the position of the global maximum, 
$G_{T}$, belongs to the zero set of $b_{H}(x)$ or to the end-points of $(-T, T)$. For this reason it should seem that the local time $l(x)=\lim _{\varepsilon \rightarrow 0} \frac{1}{2 \varepsilon} \int_{0}^{x} \mathbf{1}_{\left|b_{H}(s)\right|<\varepsilon} d s$ is the natural time scale in our problem of the maximum of $y(x)$, i.e., it is more natural to study $\tilde{y}(l)=y(x(l))$ instead of $y(x)$, where $x(l)$ is the inverse function of $l(x)$ which is continuous on the right. The process was first treated by Vergassola et al. [19] and independently used by Isozaki and Watanabe [11] to prove the Sinai asymptotics for $H=1 / 2$. It is a known fact [12] that $l(x)$ is a continuous self-similar process with parameter $h=1-H$. Consequently, $l(T)=O\left(T^{1-H}\right)$, and (15) means, roughly speaking, that $P\left(\left|\tilde{G}_{L}\right|<1\right)=L^{-1} \mathcal{L}_{L}$ where $\tilde{G}_{L}$ is the location of the maximum of $\tilde{y}(l)$ in $(-L, L)$. A more exact statement can be made. Let $\mathcal{L}_{T, i}$ be slowly varying functions that decrease as $T \rightarrow \infty$.

Statement 2. If a) $G_{T}$ and $l( \pm T)$ are weakly dependent, i.e.

$$
\left.P\left\{\left|G_{T}\right|<1,|l( \pm T)|>T^{1-H} \mathcal{L}_{T, 1}\right)\right\}>P\left(\left|G_{T}\right|<1\right) \mathcal{L}_{T, 2}
$$

and b) $P\left(\left|\tilde{G}_{L}\right|<1\right)<\left[L \mathcal{L}_{L}\right]^{-1}$, then $P\left(\left|G_{T}\right|<1\right)<\left[T^{(1-H)} \mathcal{L}_{T, 3}\right]^{-1}$.

Proof. By (16) we have

$$
\begin{aligned}
P\left(\left|G_{T}\right|<1\right) & <\mathcal{L}_{T, 2}^{-1} P\left\{\left|G_{T}\right|<1,|l( \pm T)|>T^{1-H} \mathcal{L}_{T, 1}\right. \\
\left|l\left( \pm G_{T}\right)\right| & \left.<(a \ln T)^{H}\right\}+\mathcal{L}_{T, 2}^{-1} P\left\{|l( \pm 1)|>(a \ln T)^{H}\right\}
\end{aligned}
$$

The second term on the right-hand side admits of an upper bound according to [22]: $\mathcal{L}_{T, 2}^{-1} \cdot T^{-c a}$ where $c$ is an absolute constant. It follows that (17) can be continued:

$$
\leq \mathcal{L}_{T, 2}^{-1}\left[P\left\{\left|\tilde{G}_{L(T)}\right|<(a \ln T)^{H}\right\}+O\left(T^{-a c}\right)\right],
$$

where $L(T)=T^{1-H} \mathcal{L}_{T, 1}$.

The process $y(l)$ is self-similar; therefore,

$$
\left.P\left\{\left|\tilde{G}_{L(T)}\right|<(a \ln T)^{H}\right)\right\}=P\left\{\left|\tilde{G}_{L^{*}}\right|<1\right\}<\left[L^{*} \mathcal{L}_{L^{*}}\right]^{-1},
$$

where $L^{*}=L(T)(a \ln T)^{-H}$. Combining the resulting estimates and bearing in mind that the constant " $a$ " is arbitrary, one gets Statement $2 . \diamond$

Note that the condition (b) of Statement 2 is automatically satisfied for $H=1 / 2$, because $\tilde{y}$ is a stable Levy process. The next theorem shows that this condition also holds for general self-similar processes with stationary increments (SSSi). It is the case for $H=1 / 2$ again.

Theorem 3. Let $\xi(t), \xi(0)=0$ be an SSSi-process for which sample paths have only discontinuities of the first kind, a.s. Let $M$ be $\sup \{\xi(s), s \in[0,1]\}$ and $G$ be the leftmost position of $M$ :

$$
G=\inf \left\{t \in[0,1]: \sup _{s \rightarrow t} \xi(s)=M\right\}
$$


Then

1) $G$ has a continuous probability density $\psi(t)$ in $(0,1)$, and

$$
\psi(t) \leq \psi(s) \max \left(\frac{s}{t}, \frac{1-s}{1-t}\right), \quad \forall t, s \in(0,1),
$$

i.e., $\psi \equiv 0$ or $\psi>0$.

$2)$ if $\psi \not \equiv 0$, then the position $G_{T}$ of the supremum of $\xi(t)$ in $(-T a, T(1-a))$ satisfies the following relation:

$$
P\left(\left|G_{T}\right|<1\right)=\frac{\psi(a)}{2 T}(1+o(1)), \quad T \rightarrow \infty .
$$

Remark. Theorem 3 shows that the asymptotics of type (18) is due to the presence of a nonzero distribution density for the position of the supremum of the SSSi process in $(0,1)$. That fact was first pointed out in [15] for the process $b_{H}(x)$. The proof of the general case is nearly identical with that given in [15], so it is relegated to the Appendix.

The unilateral IFBM process.
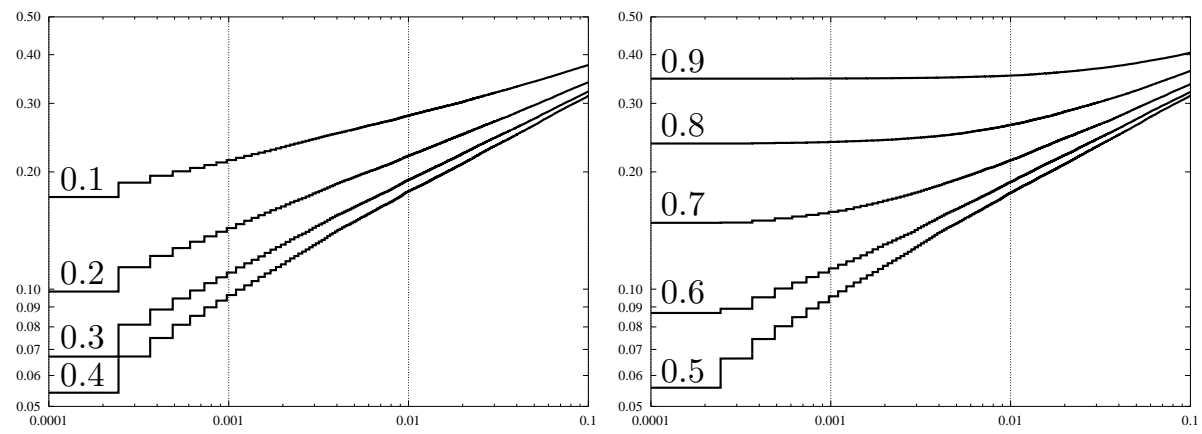

Figure 2: Distribution of the position of maximum $G$ for the IFBM process. Shown are the parts of curves related to the interval $\left(10^{-4}, 10^{-1}\right)$ together with the corresponding values of $H$

The process $y(x)=\int_{0}^{x} b_{H}(s) d s$ in $(0,1)$ was generated with the same parameters $H, T$ and $N$ as in the bilateral case. The computation is illustrated, as above, by the distribution of the position of the maximum $G$ for $y(x)$ with $H=0.1-0.4$ in Fig. 2 (left) and with $H=0.5-0.9$ in Fig. 2 (right). The plots clearly reveal the influence of the atom $p_{0}(T)$ at zero for $H>0.5$, which impedes to see the exponent $\theta=\theta(H)$ in the power law asymptotics of $F_{G}$ near zero. One can nevertheless assert that $\theta(H) \rightarrow 0$ at the endpoints $H=0$ and 1 . The maximum likelihood estimates of $\theta$ in the interval of $G: 10^{-3}-10^{-2}$ are as follows: 


$\begin{array}{ccccccc}H & : & 0.1 & 0.2 & 0.3 & 0.4 & 0.5 \\ \hat{\theta} & : & .09 & .13 & .18 & .21 & .23 \\ \theta_{0} & : & .09 & .16 & .21 & .24 & .25\end{array}$

We also list the hypothetical values of $\theta: \theta_{0}=H(1-H)$ for comparison purposes in the above table. The exact result due to Sinai [18]: $\theta=.25$ for $H=1 / 2$ shows that we can still take the error of $\hat{\theta}$ equal to 0.03 . In that case the hypothetical estimates $\tilde{y}(l)$ do not contradict the empirical ones.

Speaking in terms of the process $\tilde{y}(l)$, which can be obtained by replacing the time in $y(x)$ with local time of $b_{H}(x)$, the hypothesis $\theta_{0}(H)$ means that the position of the maximum $\tilde{G}_{L}$ in $\tilde{y}(l), 0<l<L$ has the property

$$
P\left(\tilde{G}_{L}<1\right)=L^{-H} \mathcal{L}_{L}, \quad L \rightarrow \infty
$$

where $\tilde{\mathcal{L}}_{L}$ is a slowly increasing function of $L$. In the limiting case $H \rightarrow 0$ the local time $l(x) \simeq x$, hence $\tilde{y}(l)=\xi l$ where $\xi$ is a Gaussian variate. Consequently, $P\left(\tilde{G}_{L}<1\right) \sim 1 / 2, H \rightarrow 0$, which is consistent with the hypothesis (19).

It would be natural to expect an analytical dependence of $\theta$ on $H$ for the IFBM process. Consequently, the hypothesis $\theta_{0}(H)$ can also be extended to cover the case $H>1 / 2$. That extrapolation is exact for $H=1$, because $y(x)=\xi x^{2} / 2$, where $\xi$ is a Gaussian variate, so that $P(G<x)=1 / 2$ for $H=1$. The last result corresponds to $\theta(1)=0$.

The rigorous result guarantees that $\theta(H) /(1+H)$ is decreasing for $H>1 / 2$.

Statement 4. (a) The distribution $F_{M}(x \mid H)$ for the maximum of

$$
y_{1}(t)=\sqrt{2 H+2} \int_{0}^{t} b_{H}(s) d s, \quad 0<t<1
$$

increases with increasing $H$ in the interval $(1 / 2,1)$ for any fixed $x>0$.

(b) If $F_{M}\left(x^{1+H} \mid H\right)=x^{\theta(H)} \mathcal{L}(x), x \downarrow 0$ where $\mathcal{L}(x)$ is a slowly varying function, then $\theta(H) /(1+H)$ decreases with increasing $H$ in $(1 / 2,1)$.

Proof. The process (20) differs from $y(x)$ by the normalization $E\left|y_{1}(1)\right|^{2}=1$. Let $\xi_{q}(x)=y_{1}\left(x^{\theta}\right)$ where $q=2 H+2, \theta=q_{0} / q, q_{0}=2 H_{0}+2$ and $H>H_{0}$.

Since IFBM is self-similar with parameter $h=H+1$, one has $E\left|\xi_{q}(x)\right|^{2}=$ $|x|^{q_{0}}=E\left|\xi_{q_{0}}(x)\right|^{2}$

We show in the Appendix that

$$
E \xi_{q}(x) \xi_{q}(y) \geq E \xi_{q_{0}}(x) \xi_{q_{0}}(y)
$$

when $H>1 / 2$. In that case the Slepian lemma [13] yields

$$
P\left(\max _{[01]} \xi_{q}(x)<u\right) \geq P\left(\max _{[01]} \xi_{q_{0}}(x)<u\right) .
$$


However, $\max _{[01]} \xi_{q}(x)=\max _{[01]} \sqrt{2 H+2} \int_{0}^{x} b_{H}(s) d s$ which proves the first part of the statement. The second part is an obvious corollary of the first.

\section{Conclusion}

We were testing the hypothesis that the maximum $M$ of the integral of fractional Brownian motion with index $H$ has the distribution $F_{M}\left(x^{(1+H)}\right)=$ $x^{\theta(H)} \mathcal{L}_{H}(x), x \rightarrow 0$ in $I \ni 0$, where $\mathcal{L}_{H}$ is a slowly varying function. We have presented theoretical arguments and computational evidence to support and refine the hypothesis as follows: $\theta(H)=1-H$ for $I=(-1,1)$ and $\theta(H)=$ $H(1-H)$ for $I=(0,1)$. The computational part of the problem faces the following difficulties. Due to discrete time (a step of $\Delta$ ), the analogue of $M$ in the grid case $(\tilde{M})$ has a nonzero probability $P(\tilde{M}=0)$ of the same order as $F_{M}\left(\Delta^{1+H}\right)$. It causes a slow (power law) convergence of the distributions of $\tilde{M}$ and $M$. Further, there exists an interval $\left(0, t_{0}(\Delta, H)\right), t_{0} \rightarrow 0$ as $\Delta \rightarrow 0$, where $P\left(\tilde{M}<x^{1+H}\right)$ has a power law behavior with exponent $\tilde{\theta}(H)>\theta(H)$, $H>1 / 2$. This makes the choice of the interval where $\theta(H)$ is to be estimated more difficult. When $\Delta=0.00012$ and the number of samples is $N=50000$, the accuracy of $\theta(H)$ is $\sim 0.03$ for the most favorable range of $H: H \leq 1 / 2$.

\section{Appendix}

Proof of Theorem 2. A distribution function (here $F_{G}$ ) is differentiable almost everywhere, because of monotonicity. Suppose this is true for the point $x_{0}$. Consider $x<x_{0}, \lambda=x_{0} / x>1$. The self-similarity of $\xi(x)$ yields

$$
P(G(0,1) \in d x)=P(G(0, \lambda) \in \lambda d x) \leq P(G(0,1) \in \lambda d x)=\psi\left(x_{0}\right) \frac{x_{0}}{x} d x
$$

Here, $G(a, b)$ is the leftmost position of the supremum of $\{\xi(x), x \in(a, b)\}$. Consequently, the distribution of $G(0,1)$ is absolutely continuous in $\left(0, x_{0}\right)$. Points like $x_{0}$ are dense in $(0,1)$. Consequently, $F_{G}(d x)=\psi(x) d x, x \in(0,1)$.

In virtue of $(22), \psi(x) x$ is a nondecreasing function, i.e., the discontinuities in $\psi$ are at most denumerable, while finite limits on the left and the right exist at the discontinuity points. The fact that the increments of $\xi(x)$ are stationary yields

$$
\begin{aligned}
P\{G(0,1) \in d x\} & =P\{G(-a, 1-a) \in d x-a\} \leq P\{G(0,1-a) \in d x-a\} \\
& =P\left\{G(0,1) \in d\left(\frac{x-a}{1-a}\right)\right\}
\end{aligned}
$$

for any $0<a<1$. One has

$$
\psi(x) \leq \psi\left(\frac{x-a}{1-a}\right) \frac{1}{1-a}
$$


at continuous points of $\psi$. Multiply both parts by $(1-x)$ :

$$
(1-x) \psi(x) \leq \psi\left(\frac{x-a}{1-a}\right)\left(1-\frac{x-a}{1-a}\right)=\psi(y)(1-y), \quad y=\frac{x-a}{1-a}<x .
$$

Combining both inequalities, one gets

$$
\psi(x) \leq \psi(y) \max \left(\frac{y}{x}, \frac{1-y}{1-x}\right)
$$

at all points where $x$ and $y$ are continuous. In particular, $\psi(x+0) \leq \psi(x-0) \leq$ $\psi(x+0)$, i.e., $\psi$ is continuous in $(0,1)$. If $\psi\left(x_{0}\right)=0, x_{0} \in(0,1)$, then one has $\psi(x)=0, x \in(0,1)$ from (23). Consequently, the following alternative holds: either $\psi \equiv 0$ or $\psi>0$ in $(0,1)$. The second part of Theorem 2 is an immediate corollary of the first part and the self-similarity of $\xi(x)$, see [15].

The proof of (21) in Statement 4.

Let $\xi_{q}(t)=\sqrt{q} \int_{0}^{\tau} b_{H}(s) d s, \tau=t^{\theta}, \theta=q_{0} / q<1, q=2 H+2$. The correlation function $\beta_{q}(t, s)$ of $\xi_{q}(t)$, can be written as

$$
2 t^{-q_{0}} \beta_{q}(t, s)=\frac{q_{0}}{q_{0}-\theta}\left(\rho^{\theta}+\rho^{q_{0}-\theta}\right)+\left[\left(1-\rho^{\theta}\right)^{q_{0} / \theta}-\left(1+\rho^{q_{0}}\right)\right] \frac{\theta}{q_{0}-\theta}
$$

where $\rho=s / t$. Because $\beta_{q}$ is symmetric in $t, s$, we put $\rho \leq 1$. We will show that $\beta_{q}(t, s)>\beta_{q_{0}}(t, s)$, if $q>q_{0}>3$ or, which amounts to the same thing, $H>H_{0}>1 / 2$.

We have in virtue of (24):

$2 t^{-q_{0}}\left[\beta_{q}(t, s)-\beta_{q_{0}}(t, s)\right]=\left(q-q_{0}\right)\left(1-\rho^{\theta}\right)\left(q_{0}-1\right)^{-1} R_{1}+q_{0}\left(q_{0}-1\right)^{-1} R_{2}$.

Here $\quad R_{1}=\left(1-y^{q-1}-\bar{y}^{q-1}\right)(q-1)^{-1}-\left(\bar{y}^{q_{0}-1}-\bar{y}^{q-1}\right)\left(q-q_{0}\right)^{-1}, y=\rho^{\theta}$, $\bar{y}=1-y$ and

$$
R_{2}=\int_{\theta}^{1}\left[\rho^{\alpha}-\rho^{q_{0}-\alpha}-\left(1-\rho^{\alpha}\right)^{q_{0}-1} \rho^{\alpha}\right] d \alpha \ln 1 / \rho .
$$

We now are going to show that $R_{1} \geq 0$, and $R_{2} \geq 0$, if $H \geq 1 / 2$.

Consider $R_{2}$. Put $\rho^{\alpha}=u$. Since $0<\theta<\alpha<1$ and $0<\rho<1$, it follows that $0<u<1$. The integrand in $R_{2}$ becomes

$$
\begin{aligned}
u\left[1-u^{q_{0} / \alpha-2}-(1-u)^{q_{0}-1}\right] & \geq u\left[1-u^{q_{0}-2}-(1-u)^{q_{0}-2}\right] \\
& \geq u\left[1-\max \left(1,2^{3-q_{0}}\right)\right] \geq 0
\end{aligned}
$$

The last estimate is true, because $3-q_{0}=1-2 H \leq 0$ when $H>1 / 2$. Consequently, $R_{2} \geq 0$. 
Consider $R_{1}$. The function $R_{1}(y)$ is positive around 0 and 1 :

$$
R_{1}= \begin{cases}y^{2}\left(q_{0}-1\right) / 2+O\left(y^{q-1}\right), & y \rightarrow 0 \\ \bar{y}+O\left(\bar{y}^{q_{0}-1}\right), & \bar{y} \rightarrow 0 .\end{cases}
$$

Consequently, $R_{1} \geq 0$, if the function has a single local extremum in $(0,1)$. Let $z=(1-y)^{-1} \in(1, \infty)$. Then

$$
-z^{q_{0}-2} \frac{d}{d y} R_{1}=\left[(z-1)^{q-2}-1\right]-\left[z^{q-q_{0}}\left(q_{0}-1\right)-q-1\right]\left(q-q_{0}\right)^{-1}:=f(z)
$$

We now show that $f(z)$ has a single root in $(1, \infty)$. The function

$$
f(z)= \begin{cases}-(z-1)\left(q_{0}-1\right)+O\left((z-1)^{2 H}\right), & z \rightarrow 1 \\ (z-1)^{q-2}(1+o(1)), & z \rightarrow \infty\end{cases}
$$

changes sign in $(1, \infty)$. The equation $f^{\prime}(z)=0$ or

$$
(q-2)(z-1)^{q-3}=\left(q_{0}-1\right) z^{q-q_{0}-1}
$$

determines the local extremums of $f(z)$ in $(1, \infty)$. Two strictly monotone functions occur in (27): on the left is a function that increases from 0 to $\infty$, because $q-3=2 H-1>0$, while the nonnegative function on the right decreases toward zero at infinity, because $q-q_{0}-1=2\left(H-H_{0}\right)-1<2 \cdot 1 / 2-1<0$. In that case, however, $(27)$ has the single root $1<z^{*}<\infty$. In virtue of $(26) z^{*}$ is the point of minimum, where $f\left(z^{*}\right)<0$. The function $f(z)$ is strictly increasing from $f\left(z^{*}\right)<0$ to $\infty$ in $\left(z^{*}, \infty\right)$, so that the equation $f(z)=0$ has a single root, as was to be proved.

Acknowledgements. This research was supported by the James McDonnell Foundation within the framework of the 21st Century Collaborative Action Award for Studying Complex Systems (project "Understanding and Prediction of Critical Transitions in Complex Systems"), by the National Science Foundation (grant EAR 9804859) and in part by the Russian Foundation for Basic Research (grant 99-01-00314). 


\section{REFERENCES}

1. Ammar G.S., and W.B. Cragg. Superfast solution of real positive Toeplitz systems. SIAM J. Matrix Annal. Appl., 9:1, 61-76 (1988).

2. Bardet J.M., G. Lang, A. Philippe, and M.S. Taqqu. Generators of longrange dependent processes: a survey, in: Donkham P., G. Oppenheim, and M. Taqqu (eds.), Long-range Dependence: Theory and Applications, vol. 1, 2002, 579-623, Birkhauser Production.

3. Bertoin J. The inviscid Burgers equation with Brownian initial velocity. Commun. Math. Phys. 193, 397-406 (1998).

4. Fernique X. Regularite des trajectoires des fonctions aleatoires gaussiennes. Lecture Notes in Mathematics, vol. 1480: 2-187 (1975).

5. Handa K. A remark on shocks in inviscid turbulence, in: N. Fitzmaurice et al. (eds.), Nonlinear Waves and Turbulence, pp. 339-345, 1993. Birkhauser, Boston.

6. Hu X., and S.J. Taylor. The multifractal structure of stable occupation measure. Stochastic Processes Appl. 66, 283-299 (1997).

7. Hu X., and S.J. Taylor. The multifractal structure of a general subordinator. Stochastic Processes and their Appl. 88, 245-258 (2000).

8. Jaffard S. The multifractal nature of Levy processes. Probab. Theory Relat. Fields 114:2, 207-227 (1999).

9. Isozaki Y. Asymptotic estimates for the distribution of additive functionals of Brownian motion by the Wiener-Hopf factorization method. J. Math. Kyoto Univ. 36:1, 211-227 (1996).

10. Isozaki Y., and S. Kotani. Asymptotic estimates for the first hitting time of fluctuating additive functionals of Brownian motion. Lecture Notes in Math., 1729, 374-387 (2000).

11. Isozaki Y., and S. Watanabe, An asymptotic formula for the Kolmogorov diffusion and a refinement of Sinai's estimates for the integral of Brownian motion. Proc. Japan Acad. 70A, 271-276 (1994).

12. Kahane J.-P. Some Random Series of Functions. 2nd ed., Cambridge University Press (1985).

13. Leadbetter M., G. Lindgren, H. Kootzen. Extremes and related properties of random sequences and processes. Springer-Verlag Inc. (Springer ser. in Statistics) (1986).

14. Majumdar S.N. Persistence in nonequilibrium systems. Current Science 77:3, 370-375 (1999). 
15. Molchan G. Maximum of a fractional Brownian motion: probabilities of small values. Commun. Math. Phys. 205, 97-111 (1999).

16. Molchan G.M., and Yu.I. Golosov, Gaussian stationary processes with asymptotic power spectrum. Soviet Math. Dokl. 10:1, 134-137 (1969).

17. She Z., E. Aurell, and U. Frisch. The inviscid Burgers equation with initial data of Brownian type, Commun. Math. Phys. 148, 623-642 (1992).

18. Sinai Ya.G. Statistics of shocks in solutions of the inviscid Burgers equation, Commun. Math. Phys. 148, 601-621 (1992).

19. Vergassola M., B. Dubrulle, U. Frisch, and A. Noullez. Burgers' equation, Devil's staircases and the mass distribution for large-scale structures. Astron. Astrophys. 289, 325-356 (1994).

20. Winkel M. Limit clusters in the inviscid Burgers turbulence with certain random initial velocities. J. Statistical Phys. 107, no. 3/4, 893-917 (2002).

21. Woyczynski W.A. Burgers-KPZ turbulence. Göttingen Lectures. Springer, (Lectures notes in mathematics; 1700) (1998).

22. Xiao, Y. Hölder conditions for the local times and the Hausdorff measure of the level sets of Gaussian random fields. Probab. Theory Relat. Fields, 109, 129-157 (1997). 